\title{
A high-density SNP genotyping array for Brassica napus and its ancestral diploid species based on optimised selection of single-locus markers in the allotetraploid genome
}

\author{
Wayne E. Clarke ${ }^{1} \cdot$ Erin E. Higgins $^{1} \cdot$ Joerg Plieske $^{2} \cdot$ Ralf Wieseke $^{2} \cdot$ Christine Sidebottom $^{3} \cdot$ Yogendra Khedikar $^{1} \cdot$

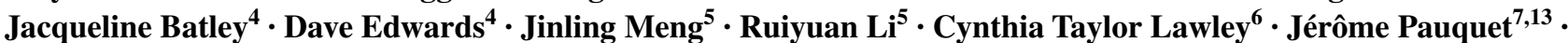 \\ Benjamin Laga $^{8} \cdot$ Wing Cheung $^{9} \cdot$ Federico Iniguez-Luy ${ }^{10} \cdot$ Emmanuelle Dyrszka $^{11} \cdot$ Stephen Rae $^{8}$. \\ Benjamin Stich $^{12} \cdot$ Rod J. Snowdon $^{14} \cdot$ Andrew G. Sharpe $^{3} \cdot$ Martin W. Ganal $^{2} \cdot$ Isobel A. P. Parkin $^{1}$
}

Received: 26 April 2016 / Accepted: 18 June 2016 / Published online: 30 June 2016

(C) The Author(s) 2016. This article is published with open access at Springerlink.com

\begin{abstract}
Key message The Brassica napus Illumina array provides genome-wide markers linked to the available genome sequence, a significant tool for genetic analyses of the allotetraploid $B$. napus and its progenitor diploid genomes.

Abstract A high-density single nucleotide polymorphism (SNP) Illumina Infinium array, containing 52,157 markers, was developed for the allotetraploid Brassica napus. A stringent selection process employing the short probe sequence for each SNP assay was used to limit the majority of the selected markers to those represented a minimum
\end{abstract}

Communicated by C. F. Quiros.

Electronic supplementary material The online version of this article (doi:10.1007/s00122-016-2746-7) contains supplementary material, which is available to authorized users.

Isobel A. P. Parkin

isobel.parkin@agr.gc.ca

1 Agriculture and Agri-Food Canada, 107 Science Place, Saskatoon, SK S7N 0X2, Canada

2 TraitGenetics GmbH, Am Schwabeplan 1b, Stadt Seeland OT, 06466 Gatersleben, Germany

3 National Research Council Canada, 110 Gymnasium Place, Saskatoon S7N 0W9, Canada

4 School of Plant Biology and The UWA Institute of Agriculture, The University of Western Australia, 35 Stirling Highway, Crawley, Perth 6009, Australia

5 National Key Laboratory of Crop Genetic Improvement, Key Laboratory of Rapeseed Genetic Improvement, Ministry of Agriculture P. R. China, Huazhong Agricultural University, Wuhan 430070, China

6 Illumina, Inc., 9885 Towne Centre Drive, San Diego, CA 92121, USA number of times across the highly replicated genome. As a result approximately $60 \%$ of the SNP assays display genome-specificity, resolving as three clearly separated clusters (AA, $\mathrm{AB}$, and $\mathrm{BB}$ ) when tested with a diverse range of $B$. napus material. This genome specificity was supported by the analysis of the diploid ancestors of $B$. napus, whereby 26,504 and 29,720 markers were scorable in B. oleracea and B. rapa, respectively. Forty-four percent of the assayed loci on the array were genetically mapped in a single doubled-haploid $B$. napus population allowing alignment of their physical and genetic coordinates. Although strong conservation of the two positions was shown, at least $3 \%$ of the loci were genetically mapped to a homoeologous position compared to their presumed physical position in the respective genome, underlying the importance of genetic corroboration of locus identity. In

7 BIOGEMMA 6, chemin des Panedautes, 31700 Mondonville, France

8 Bayer BioScience NV, 9052 Ghent, Belgium

9 DNA Landmarks Inc, 84 Rue Richelieu, St-Jean-sur-Richelieu, QC J3B 6X3, Canada

10 Genomics and Bioinformatics Unit, Agri Aquaculture Nutritional Genomic Center (CGNA), Conicyt-Regional, Gore La Araucania, R10C1001, Temuco, Chile

11 Syngenta France SAS, 12 Chemin de l'hobit, B.P. 27, 31790 Saint-Sauveur, France

12 Max Planck Institute for Plant Breeding Research, Carl-von-Linné-Weg 10, 50829 Cologne, Germany

13 Present Address: SYNGENTA France SAS, 346, route des Pasquiers, 84260 Sarrians, France

14 Department of Plant Breeding, IFZ Research Centre for Biosystems, Land Use and Nutrition, Justus Liebig University, Giessen, Germany 
addition, the alignments identified multiple rearrangements between the diploid and tetraploid Brassica genomes. Although mostly attributed to genome assembly errors, some are likely evidence of rearrangements that occurred since the hybridisation of the progenitor genomes in the $B$. napus nucleus. Based on estimates for linkage disequilibrium decay, the array is a valuable tool for genetic fine mapping and genome-wide association studies in B. napus and its progenitor genomes.

\section{Introduction}

Brassica napus is an economically important oilseed crop that is primarily grown to extract the healthy edible oil from the seed, but it is now also grown as a renewable feedstock for biodiesel. In addition, there are vegetable types of the species that have been bred for both human and animal consumption. It is a temperate crop widely grown in both the Northern and Southern hemispheres due to available genotypic variation for flowering time and response to photoperiod. It is believed that $B$. napus emerged from a small number of hybridisation events between the diploid progenitors Brassica oleracea (C genome) and Brassica rapa (A genome) (UN 1935) that probably occurred in the southern Mediterranean and possibly regions of Asia around 7000 10,000 years ago (Chalhoub et al. 2014). The progenitors, B. oleracea and B. rapa, are also important predominantly vegetable crop species that each display a wide range of genetic and morphological diversity (Dixon 2006).

There are extensive worldwide breeding efforts in $B$. napus and its diploid relatives in both the public and private domains that contribute to developing higher value crops with improved yields (Iniguez-Luy and Federico 2011; Snowdon and Iniguez Luy 2012). Such breeding efforts are benefiting from access to a burgeoning collection of genetic and genomic resources for the Brassica species culminating in the recent release of the diploid and amphidiploid genomes that complete one axis of U's triangle and define the B. napus genome (Chalhoub et al. 2014; Parkin et al. 2014; Wang et al. 2011). The now available genome sequences can be exploited to identify candidate genes for traits of interest, but their primary utility in breeding is in the development of genetic markers for marker assisted selection and, more recently, genomic selection. Genomic selection or predictive breeding is showing potential for application in crop species, where traits can be controlled by multiple small-effect QTLs, as more sophisticated algorithms have been developed to overcome the statistical challenges of working with disproportionately larger numbers of marker loci than samples tested (Jannink et al. 2010).

The availability of genome sequences and access to relatively economical next-generation sequencing technologies has provided the impetus to identify extensive nucleotide variation among different plant species. The abundance of single nucleotide polymorphisms (SNPs) across plant genomes has made them highly desirable for marker development (Ganal et al. 2009, 2012). High-throughput (tens of thousands or higher) SNP screening can be achieved effectively by either genotyping-by-sequencing (GBS) or high-density SNP arrays. GBS requires no former knowledge of available SNPs within a species, but is heavily reliant on bioinformatics capacity, and although common SNP will be found across experiments, the SNP profile identified is dependent on the genotypes queried (Deschamps et al. 2012). In comparison, high-density SNP arrays provide a common platform that can be continuously used and replicated across multiple labs with minimal computational requirement (Ganal et al. 2012). However, such SNP genotyping arrays involve significant development costs to identify sufficient numbers of robust, informative loci that fulfill assay design criteria. Identifying high-quality SNP loci for array design requires sequence data from sufficient numbers of genotypes to be able to assess polymorphism levels and associated allele ratios across the diversity of a species to minimise ascertainment bias. In addition, genome duplication in polyploid genomes, such as B. napus, confounds the design of SNP assays, since nucleotide variation among closely related orthologous or paralogous sequences is often misinterpreted as allelic variation (Parkin et al. 2010). Furthermore, since the SNPs are evaluated through hybridisation, multiple homologous and homoeologous loci may hybridise to a single SNP oligonucleotide probe leading to highly compressed and often irresolvable SNP patterns.

The current manuscript describes the development of a high-density $(>50,000)$ Illumina Infinium ${ }^{\circledR}$ SNP array designed for genotyping in $B$. napus, that can also be applied to the diploids, B. oleracea and B. rapa. Next-generation sequence data from both genomic and transcriptome sources were utilised to identify millions of preliminary SNP loci across the B. napus genome. Extensive filtering of these data led to the development of a highly effective tool for Brassica breeding with the majority of the SNP assays targeting single loci within the amphidiploid genome. The efficacy of the array was tested through the generation of cluster files, which define common allele clusters across a range of genotypes in all three species, and a high-density genetic map for B. napus.

\section{Materials and methods}

\section{Reference mapping and variant calling}

Pseudo-genome sequences of the diploid $\mathrm{A}$ and $\mathrm{C}$ genomes (283.8 and $488.6 \mathrm{Mb}$, respectively) were combined into a single reference sequence set for mapping. Sequence reads 
from each genotype were aligned independently using the CLC Genomics Server v3.6. Default parameters for the mapping algorithm were used except for the mapping identity parameter which was increased to $98 \%$ to facilitate resolution of homoeologous sequence reads. Mapped reads were interrogated for sequence variation using the CLC Genomics Server v3.6 variant discovery algorithm. A minimum depth of coverage of $3 \times$ for 454 and $8 \times$ for Illumina data was required for SNP calling. Mapping data and variant calls were exported from CLC in the form of SAM alignment files and tab-delimited text files, respectively. Data from these files were combined using a custom Perl script to determine a missing, reference, or variant call in each genotype at each covered position of the genome.

\section{SNP filtering}

Combined SNP results were filtered using custom Perl scripts and eliminated based on the following criteria: (1) SNP positions without suitable flanking sequence ( $60 \mathrm{bp}$ on at least one side of the SNP with no variation); (2) SNP positions with more than two variations within the surveyed genotypes; (3) SNP positions with high levels of heterozygous calls, biased allele ratio, or missing data; (4) Illumina Assay Design Tool (ADT) score less than 0.6; (5) SNP positions where the variation was the result of a transversion.

\section{Probe matching and SNP selection}

Probe sequences for all filtered SNPs were obtained from Illumina and then aligned to the reference sequences using the open source alignment tool BLAT with default parameters (Kent 2002). These alignments were parsed using a custom Perl script to determine the number of times the probe sequence from a particular SNP matched to the reference sequence set. A probe alignment was considered to be matched if 35 consecutive base pairs of the probe were fully aligned. SNPs were ranked based on the number of times their probe sequence matched the reference sequence set and SNPs with fewer probe matches preferentially selected.

\section{Experimental SNP data collection}

The cluster file for B. napus was generated at AAFC through analysis of 437 genotypes and at TraitGenetics through the analysis of 432 genotypes. The cluster files for B. oleracea and B. rapa were generated with 129 and 121 samples, respectively. In both laboratories, DNA was extracted from young leaf tissue of greenhouse grown plants using a cetyltrimethylammonium bromide (CTAB)based method (Murray and Thompson 1980). DNA was quantified and $200 \mathrm{ng}$ were hybridised to the Brassica $60 \mathrm{~K}$ Infinium array as described in the manufacturer's protocol (Illumina Inc., San Diego, CA). The arrays were scanned using an Illumina HiScan or BeadArray Reader, and SNP data were analysed using the Genotyping module of the GenomeStudio software package with the setting for the No Call threshold set to 0.05 .

\section{Generation of the genetic map}

DNA from 124 lines of a doubled-haploid (DH) population (derived from a cross between DH12075 and PSA12 and named SG DH, Parkin et al, unpublished) was hybridised to the Brassica $60 \mathrm{~K}$ Infinium array, and allele calls were made using the newly generated cluster file. The genetic linkage map was generated using the MSTmap software package (Wu et al. 2008). The map order was checked manually to ensure the optimal placement of the SNP loci, and a bin map was generated. Final-map distances were calculated using the Kosambi mapping function and the Mapmaker v3 software (Lander et al. 1987).

\section{Results}

\section{Array design}

A set of 54,866 SNP assays, previously identified and tested on the Illumina platform, were provided from a number of different sources (Bus et al. 2012; Dalton-Morgan et al. 2014) (Cheung, Dryszka, Laga, Pauquet, Rae, unpublished data). The remainder of the SNP assays that were used in the array design was processed using a single pipeline (Supplementary Figure 1). Next-generation sequencing data were collated from two previously published data sets described in Harper et al. (2012), which contributed RNASeq data from 42 different B. napus genotypes, and Clarke et al. (2013), which contributed Illumina and Roche 454 sequence capture data from nine $B$. napus genotypes. In addition, Roche $454(1.16 \mathrm{~Gb})$ data from genomic material and Illumina HiSeq $(417.85 \mathrm{~Gb})$ data from both genomic and transcriptome sources were generated for an additional 13 B. napus, four B. oleracea, and three B. rapa genotypes (Supplementary Table 1).

The array was designed prior to the release of the $B$. napus genome sequence (Chalhoub et al. 2014). Thus, high-quality sequence reads were reference mapped using CLC Genomics Server v3.6 to a pseudo B. napus genome derived from concatenating the genome sequences of B. rapa (Wang et al. 2011) and B. oleracea (Parkin et al. 2014). Considering only uniquely matching reads, over $570 \mathrm{~Gb}$ of sequence data were aligned to the pseudogenome providing an estimated $738 \times$ depth coverage, 
Table 1 SNPs and filtering steps used for array design

\begin{tabular}{lrr}
\hline Filter step & SNPs excluded & SNP count \\
\hline None & 0 & $24,528,374$ \\
Flanking sequence & $18,619,172$ & $5,909,202$ \\
Multi-allele SNP $^{\text {Confidence }}$ & 7671 & $5,901,531$ \\
Illumina ADT score $(<0.6)_{\text {Transversions }}^{5,742,443}$ & 159,088 \\
& 33,556 & 125,532 \\
\hline
\end{tabular}

a SNP positions were filtered for high levels of heterozygous calls, biased allele ratio, or missing data as described in the Results section

although it should be noted that the inclusion of transcriptome data can bias the overall distribution with over-representation of some genic regions. SNP calling was completed using the SNP Discovery algorithm of the CLC Genomics Server, and all relevant data were exported for further filtering. Custom Perl scripts were used to generate an output file that included the SNP id, reference id, and position, flanking sequence where available, the reference allele, and for each individual surveyed, the SNP call, depth, and frequency data. These data were then filtered in three steps. First, SNPs were excluded if there was insufficient SNP-free flanking sequence (60 bp on at least one side). This step removed the largest number of the identified SNP loci (76 \%) (Table 1). In the second step, SNPs were excluded if they were multi-allelic (more than two alleles), since these cannot be efficiently assayed using the Illumina platform. The final step identified high-confidence SNP loci, SNPs were excluded when the frequency of individuals with missing data was greater than $70 \%$, the frequency of individuals that showed heterozygous calls was greater than $40 \%$, and finally, if the allele frequency was higher than 0.8 or lower than 0.2 . Table 1 shows the attrition at each filtering step. A final set of 180,398 SNP loci consisting of filtered and previously tested SNPs were submitted to the Illumina Assay Design Tool, which returned 161,917 SNPs with a minimum recommended score at or above 0.6 .

The specificity of each Illumina SNP assay is reliant on a single 50 bp probe sequence flanking one side of the SNP, the length of which can lead to ambiguous matching across genomes with any level of redundancy. To filter potential designs to reduce the impact of high copy probe sequences, the $50 \mathrm{bp}$ probes for each possible assay design were matched using BLAT to the pseudo-genome. More than half (74 \%) of the filtered SNP assays had probe sequences that mapped to multiple regions of the Brassica genome. The final SNP list submitted for Illumina bead design contained 15,141 previously tested SNP loci, 32,294 newly designed SNP loci that matched the pseudo-genome uniquely, and 11,029 SNP loci that matched twice. Once
Fig. 1 GenomeStudio images showing representative SNP cluster patterns across Brassica napus genotypes. One cluster representing one parental allele is coloured in red (AA), the second in blue (BB), and heterozygote genotypes in purple. a Shows a genome-specific SNP marker in B. napus, almost $60 \%$ of the SNP loci show this clear separation of the expected three genotypes. b SNP locus likely resulting from hybridisation of two segregating homoeologous loci reveals five clusters and an excess of heterozygotes. c and d Show a SNP locus called automatically by the software and after manual adjustment of the cluster profile, respectively. e SNP locus where one parental allele shows no hybridisation (presence/absence marker)

synthesized, 52,157 SNP markers on the Brassica $60 \mathrm{~K}$ array passed bead representation and decoding quality metrics, including $1213 \mathrm{~A} / \mathrm{T}$ or $\mathrm{C} / \mathrm{G}$ SNPs, which are represented by Infinium I bead types that require two beads per assay.

\section{Cluster file generation for reliable scoring of the SNP markers in Brassica napus and its diploid ancestors}

The most efficient high-throughput application of an Illumina array can be achieved with the development of a robust cluster file that defines the expected intensity level of the three genotype classes $(\mathrm{AA}, \mathrm{AB}$, and $\mathrm{BB}$ ) for each SNP locus. The cluster file is applied to intensity data to automatically call the genotypes for experimental samples, thus allowing easy comparison of data across labs (Fig. 1a). At AAFC Saskatoon, the first data set included 327 B. napus genotypes of both annual and biennial types, from diverse origins, ten $F_{1}$ lines, and a subset of lines from two DH mapping populations. Independently at TraitGenetics, a second data set was generated that consisted of 432 mostly winter-type B. napus genotypes, including 67 hybrids, 88 $\mathrm{F}_{2}$, and 20 resynthesized B. napus lines. The two data sets were analysed independently and the resultant cluster files compared. After filtering out 173 SNP from the cluster file that displayed low intensity across the majority of the samples, 51,984 SNP remained. Based on the genotypes tested at AAFC, 1678 loci were monomorphic and for the genotypes tested at TraitGenetics, 2444 markers were monomorphic. Due to the strong sequence similarity between the $\mathrm{A}$ and $\mathrm{C}$ genomes of B. napus, it was anticipated that some of the SNP loci would display cluster patterns reflecting co-hybridisation of homoeologous loci. In such instances, when both homoeologous loci are polymorphic, the resultant SNP patterns are not automatically resolvable, generally these result in four-to-five clusters, and the Illumina software will identify exceptionally high numbers of heterozygotes (Fig. 1b). However, when one of the homoeologous loci is monomorphic, the genotype cluster intensities are shifted to one side of the theta space (actual genotype would be, for example, AAAA, AAAB, and AABB), leading to false cluster assignment with the routine analysis tools (Fig. 1c); yet, the cluster definition of such an SNP 
(a)
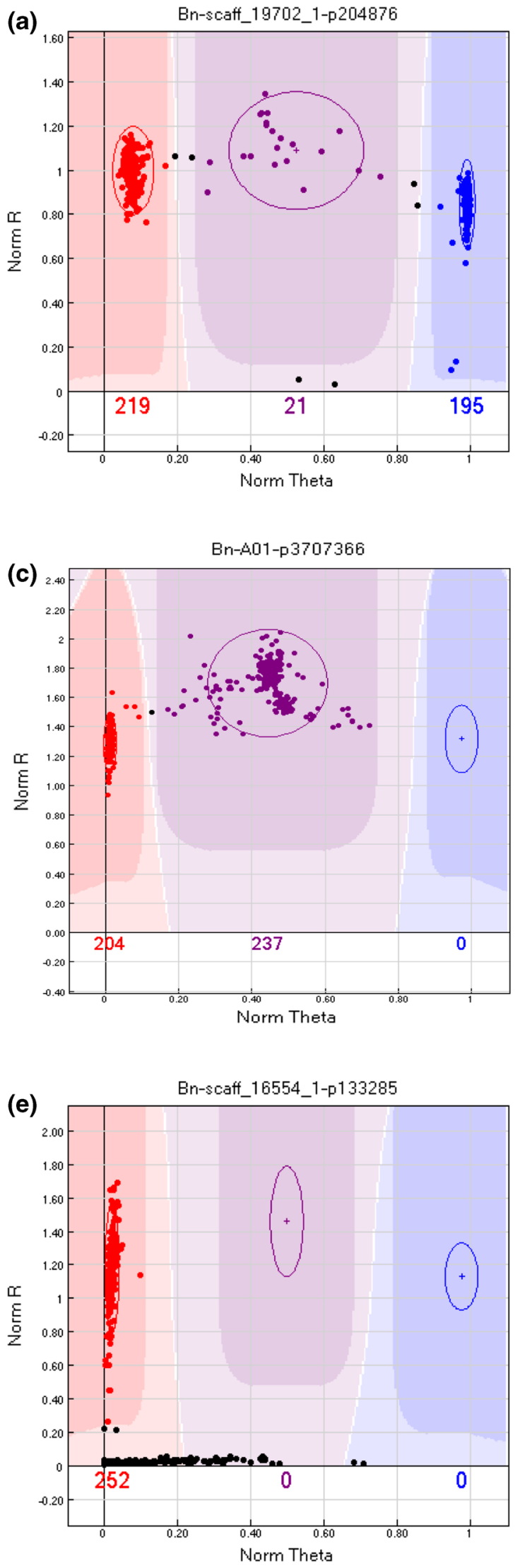

(b)
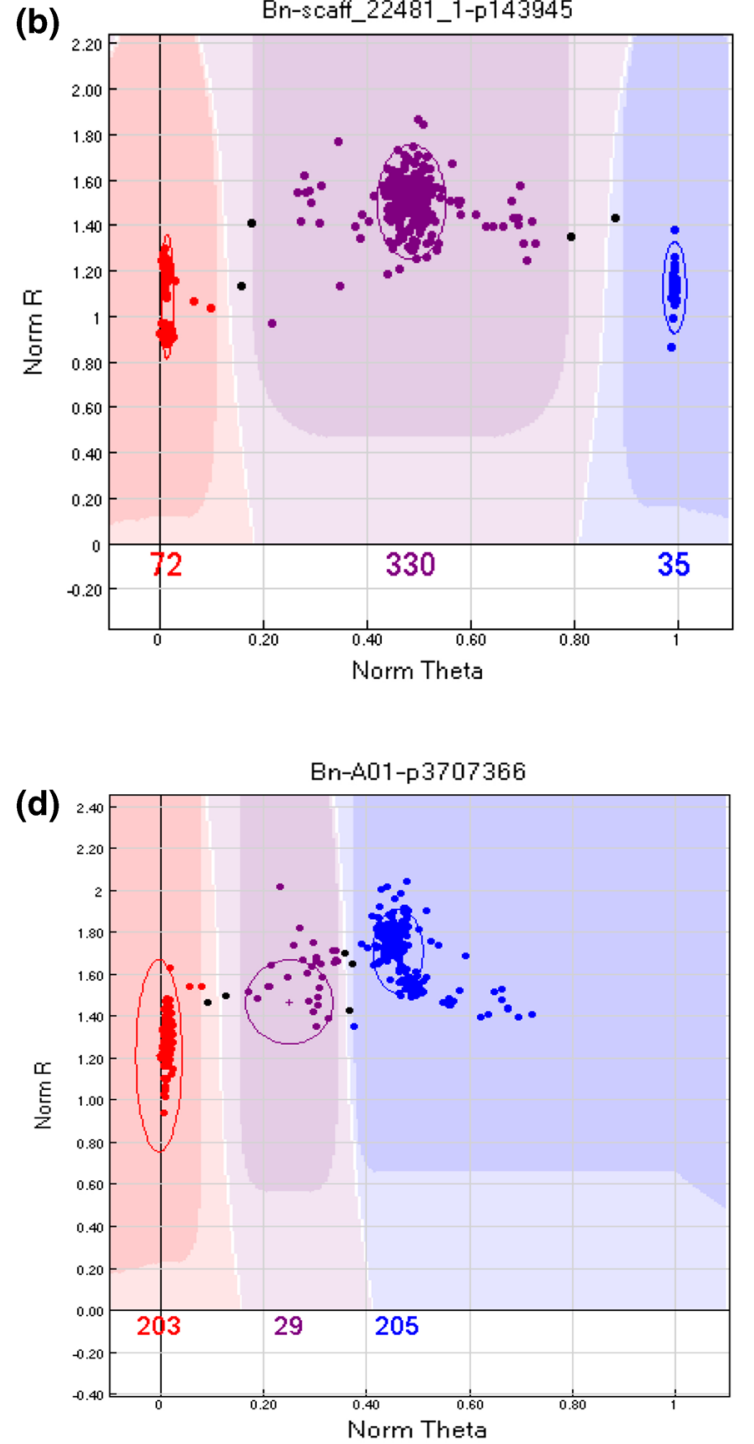
locus can be optimised manually to reflect the correct genotype positions, rendering it perfectly scorable (Fig. 1d). The two labs independently assayed for such loci, and manually adjusted the cluster assignments where necessary. By assessing the number of polymorphic markers that showed a cluster pattern that was indicative of a single copy locus, with three possible allelic states (homozygous allele AA, heterozygous $\mathrm{AB}$, and homozygous allele $\mathrm{BB}$ ) distributed over the entire theta space (difference between mean AA $\theta$ and mean BB $\theta>0.6$ ), between 34,248 (TraitGenetics) and 37,536 (AAFC Saskatoon) loci were determined to be effectively genome specific. For a small number of markers, fluorescence was observed for only one allele, which could be informative in certain populations, but would be unable to detect heterozygous individuals (Fig. 1e). In total, 47,304 markers were defined as scorable and are retained in the current $B$. napus cluster file.

Diploid samples included in the initial analysis indicated that a subset of the loci could not be scored accurately in the diploids using the B. napus cluster file (Fig. 2a-c). To facilitate the use of the Brassica array for scoring in the diploid ancestors, 129 B. oleracea lines, hybrids and representative segregating material, and $121 \mathrm{~B}$. rapa lines, $\mathrm{F}_{1} \mathrm{~s}$ and representative segregating material were analysed with the array. Based on these results, cluster files for the two ancestral species were generated, mainly based on modified cluster positions for those markers that were not genome specific. The final cluster file for B. oleracea contained 26,504 scorable markers of which 21,113 were polymorphic in the investigated material, and the $B$. rapa cluster file contained 29,720 scorable markers of which 22,695 were polymorphic in the investigated material (Supplementary Table 2).

\section{Physical and genetic positions of SNPs on Brassica genomes}

The physical positions of the assayed loci in the diploid A and $\mathrm{C}$ genomes were determined during the SNP calling process, based on unique read mapping to the reference sequence set, consisting of the A genome of $B$. rapa (Wang et al. 2011) and the $C$ genome of $B$. oleracea (Parkin et al. 2014). The physical position was also determined in two recently completed $B$. napus genomes, one a winter type (Chalhoub et al. 2014) and the second a spring type (Parkin et al. unpublished), by aligning the DNA flanking each of the SNP loci to each genome using BLAT (Kent 2002). The best hit and associated percent identity of the match for each genome were then extracted from the BLAT results. Based on a percent identity of at least $85 \%, 50,255$ SNPs were positioned in the spring-type genome sequence and 49,794 were positioned in the winter-type genome sequence. Taking both genome sequences together, a total of 51,172 SNPs could be matched to one or both B. napus genomes (Fig. 3).
It was recognized that the length of the query sequence could lead to ambiguities or erroneous matches due to the highly redundant nature of each genome. The latter would be particularly true for matches to the B. napus genome, where in addition to the strong homology between the two constituent genomes, there are also regions of effective identity resulting from homoeologous exchanges between the $\mathrm{A}$ and $\mathrm{C}$ genomes (Chalhoub et al. 2014). Based on the BLAT scores, 22,258 and 23,191 SNPs could be unambiguously positioned on the $\mathrm{A}$ and $\mathrm{C}$ genomes, respectively, while 2138 were placed on either the $\mathrm{A}$ or $\mathrm{C}$ with equal probability (Supplementary Table 3). In addition, 4570 SNPs could not be positioned on the pseudo-chromosomes as a result of either missing data in B. napus or the alignment of the SNP sequence to an unanchored scaffold in one or both B. napus genomes. The SNP loci were largely found in non-coding regions, although 17,955 lay within annotated gene sequences, only 8681 of which were positioned within an exon (Supplementary Table 4).

To genetically position 21,766 (46\%) of the SNP loci, the highly polymorphic SG DH population derived from a cross between a resynthesized B. napus and an established B. napus line was used (Supplementary Tables 5 and 6). Based on informative recombination events, these loci were placed in 1310 bins across the 19 linkage groups and covered a length of $1815 \mathrm{cM}$ (Table 2). The loci were distributed with on average one marker every $0.15 \mathrm{cM}$ or less (Table 2). There were a number of genetically defined bins with a higher than average density of markers, which tended to cluster together and were associated with regions of low recombination, predominantly found in the vicinity of the presumed centromeric regions (Fig. 3).

Only markers that were positioned both genetically and physically could be definitively positioned on the $B$. napus genome. In general, there was good correspondence between the two, with 20,138 of the 21,766 (93\%) SNP loci genetically mapping to the position expected based on sequence alignment. In addition, $3 \%$ of loci that were physically mapped to one position were genetically mapped to the homoeologous region of the genome (Fig. 4). The remaining $4 \%$ did not match either the expected physical position or the homoeologous position. Some of these loci were genetically mapped to unanchored B. napus scaffolds, but others mapped to a different position in B. napus than expected based on the diploid genome from which the SNP probe was designed, suggesting inconsistencies with the original assemblies. This was particularly true for the $B$. rapa genome where genomic regions of varying sizes $(0.1-1.1 \mathrm{Mb})$ were found to be anchored to the wrong chromosome relative to the two B. napus genome sequences (Supplementary Table 3 ). This is likely due to the relatively low marker density that was used to anchor the B. rapa genome assembly (Wang et al. 2011). 
Fig. 2 GenomeStudio images showing representative SNP cluster patterns in the different Brassica species for the marker Bn-scaff_23108_p362932. One parental allele is coloured in red (AA), the second in blue (BB), and heterozygotes in purple.

The SNP marker is polymorphic, but not genome-specific in B. napus, a resulting in condensed clusters due to the detection of the homoeologous locus on the other genome. In the diploid $B$. rapa, $\mathbf{b}$ this marker is polymorphic and shows widely distributed clusters (no second homoeologous locus detected, typical diploid pattern). In $B$. oleracea material, this marker is monomorphic

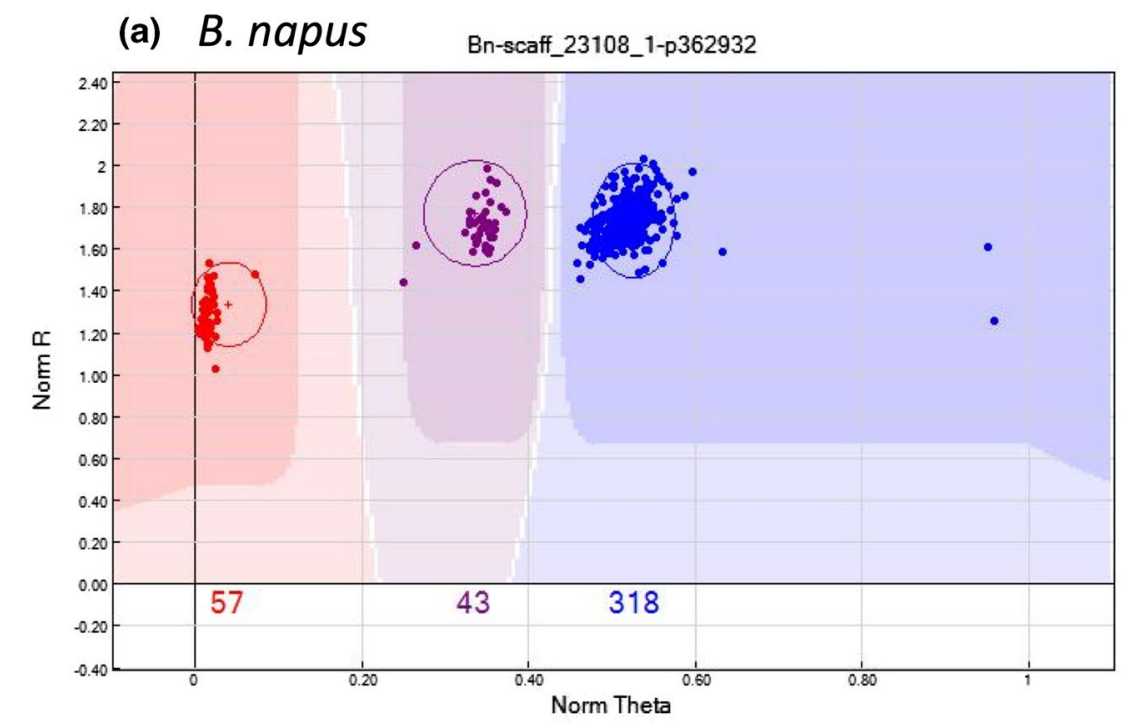

(b) B. rapa

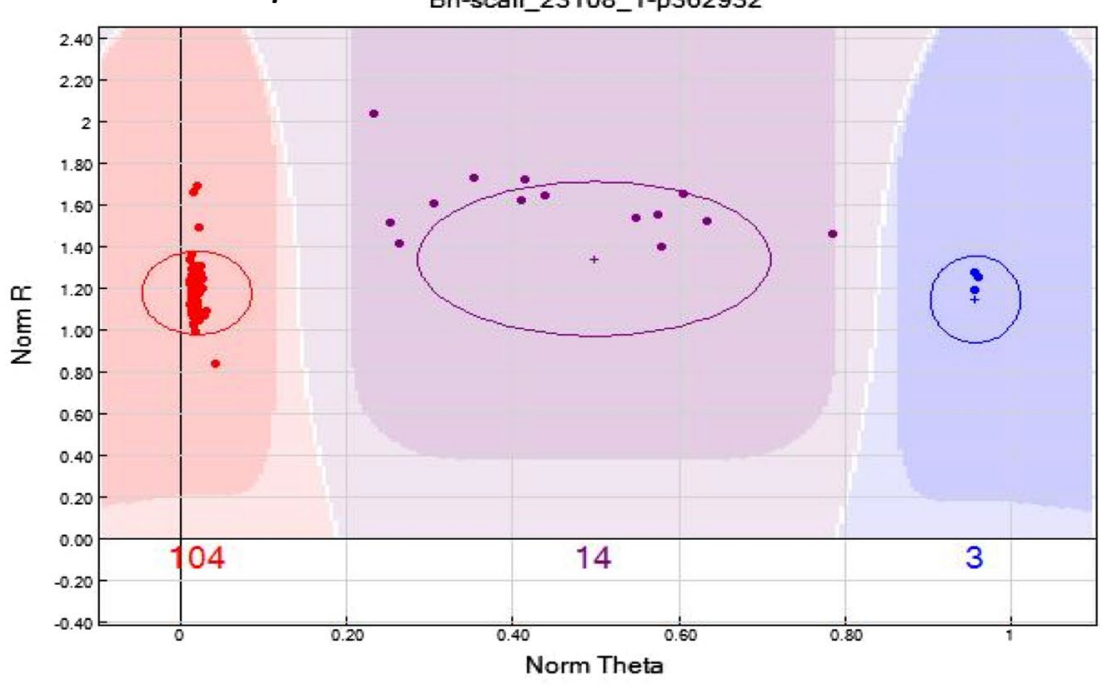

(c) B. oleracea

Bn-scaff_23108_1-p362932

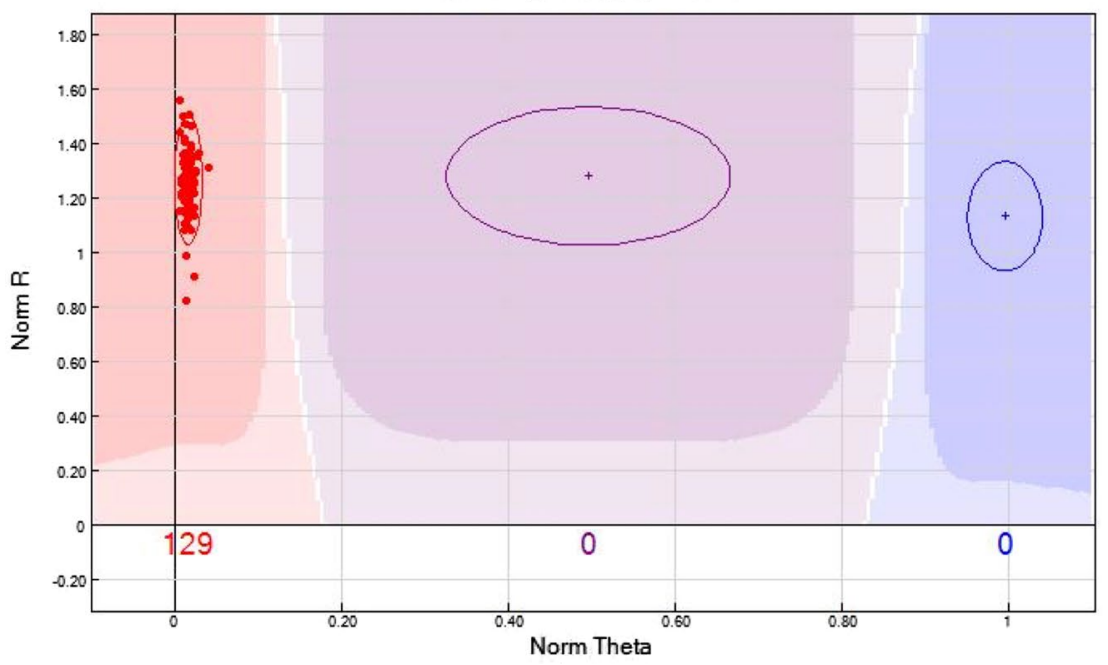




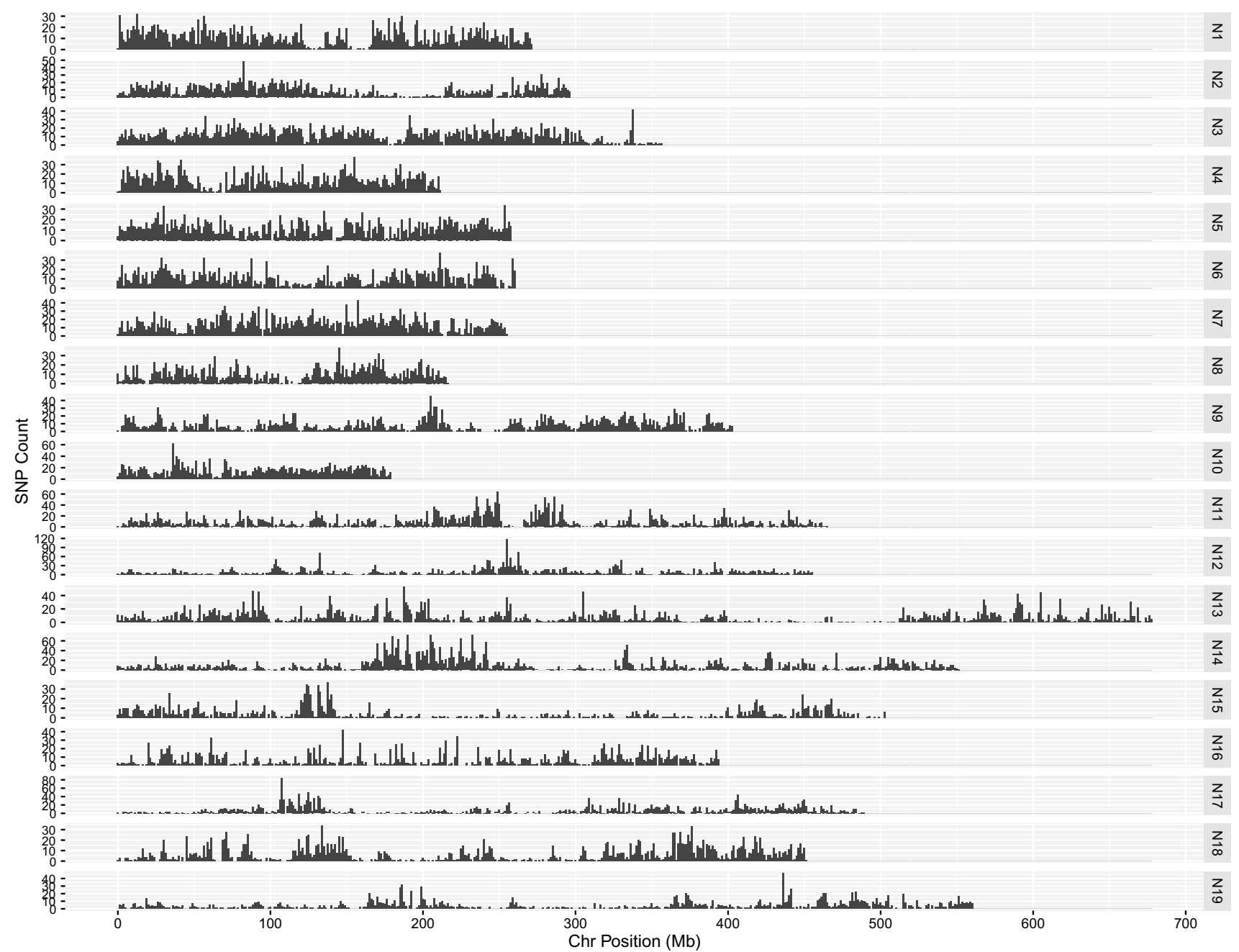

Fig. 3 Physical distribution of SNP loci across the B. napus genome. The SNP loci were aligned to the genome of spring-type DH12075 based on BLAT scores, with the numbers of SNP loci per $125 \mathrm{~Kb}$ window indicated on the $y$ axis for each chromosome

It is possible that some differences between the genomes could reflect true chromosomal rearrangements; indeed, the relatively large inversion found to differentiate both ends of A10 from N10, could be evidence of such an event, since it appears common to both B. napus genotypes (Fig. 5). However, a similar but smaller inversion at the top of A7/N7 is specific to only the Darmor bzh B. napus genotype (Supplementary Table 3). The physical and genetic positions of the SNP loci have been imported into a web tool that visualizes these alignments (http://aafc-aac.usask.ca/Bn60). Particular regions of the genome can be selected to identify potentially useful SNP loci in any region of interest.

\section{Discussion}

The recent release of three Brassica crop genome sequences has provided opportunities for the development and application of new breeding tools. The complexity of the $B$. napus genome becomes evident when carrying out genetic mapping even for relatively simple quality-based traits, where multiple loci control their expression. To dissect and follow genetically, complex traits within Brassica breeding programs robust sequence-based markers are required. The high-throughput genotyping array described here offers an excellent platform for facilitating such analyses and allows ready access to a set of well-characterised markers.

The SNP pipeline used to develop the majority (approximately $74 \%$ ) of the assays on the Brassica array included a number of steps to limit the impact of genome duplication and allopolyploidy on the resultant design. Reference mapping of short-read sequences to the constituent diploid genomes was optimised to prevent matching to homoeologous regions, sequence variation was avoided in the immediate proximity of the target SNP, and regions where multiple alleles were identified were excluded, all limiting the 
Table 2 Distribution of genetically and physically positioned SNP loci in the B. napus genome

\begin{tabular}{|c|c|c|c|c|c|c|c|c|}
\hline Linkage group & $\begin{array}{l}\text { Number of } \\
\text { genetically } \\
\text { defined bins }{ }^{\mathrm{a}}\end{array}$ & $\begin{array}{l}\text { Number of } \\
\text { physically } \\
\text { positioned SNP } \\
\text { loci }^{\text {b }}\end{array}$ & $\begin{array}{l}\text { Physical length } \\
\text { of pseudo-mol- } \\
\text { ecule }(\mathrm{Kb})\end{array}$ & $\begin{array}{l}\text { Number of } \\
\text { mapped SNP } \\
\text { loci }\end{array}$ & $\begin{array}{l}\text { Map distance } \\
(\mathrm{cM})\end{array}$ & Markers/cM & $\mathrm{CM} /$ marker & $\mathrm{Kb} /$ marker \\
\hline A1 & 60 & 2672 & 27,105 & 913 & 75.9 & 12.03 & 0.08 & 10.19 \\
\hline $\mathrm{A} 2$ & 74 & 2425 & 29,627 & 946 & 98.2 & 9.63 & 0.10 & 12.22 \\
\hline A3 & 94 & 3185 & 35,753 & 1330 & 114.3 & 11.64 & 0.09 & 11.23 \\
\hline A4 & 50 & 2112 & 21,080 & 1085 & 57.6 & 18.84 & 0.05 & 9.98 \\
\hline A5 & 78 & 2332 & 25,706 & 1121 & 99.6 & 11.26 & 0.09 & 11.06 \\
\hline A6 & 89 & 2302 & 26,146 & 1019 & 100.1 & 10.18 & 0.10 & 11.32 \\
\hline A7 & 46 & 2529 & 25,458 & 1333 & 60 & 22.52 & 0.04 & 10.07 \\
\hline A8 & 55 & 1863 & 21,685 & 953 & 85 & 11.21 & 0.09 & 11.64 \\
\hline A9 & 89 & 2452 & 40,546 & 1279 & 127.5 & 10.03 & 0.10 & 16.43 \\
\hline A10 & 63 & 2053 & 17,911 & 841 & 75.6 & 11.12 & 0.09 & 8.72 \\
\hline $\mathrm{C} 1$ & 46 & 3418 & 45,604 & 1882 & 71.3 & 26.40 & 0.04 & 13.65 \\
\hline $\mathrm{C} 2$ & 47 & 3743 & 47,311 & 1241 & 69.8 & 17.78 & 0.06 & 12.20 \\
\hline $\mathrm{C} 3$ & 118 & 3870 & 67,777 & 1804 & 165.3 & 10.91 & 0.09 & 17.51 \\
\hline $\mathrm{C} 4$ & 88 & 4399 & 55,069 & 1443 & 136.9 & 10.54 & 0.09 & 12.55 \\
\hline $\mathrm{C} 5$ & 65 & 1600 & 48,717 & 651 & 124.4 & 5.23 & 0.19 & 31.46 \\
\hline C6 & 40 & 1982 & 40,797 & 980 & 42.6 & 23.00 & 0.04 & 19.88 \\
\hline $\mathrm{C} 7$ & 82 & 2784 & 48,823 & 1360 & 101.7 & 13.37 & 0.07 & 17.54 \\
\hline $\mathrm{C} 8$ & 68 & 2151 & 44,716 & 742 & 104 & 7.13 & 0.14 & 20.97 \\
\hline C9 & 58 & 1782 & 55,995 & 843 & 106 & 7.95 & 0.13 & 31.42 \\
\hline Total & 1310 & 49,744 & 725,833 & 21,766 & 1814.9 & 11.99 & 0.08 & 14.59 \\
\hline
\end{tabular}

a The genetic map position is based on mapping data from the SG DH population

b The physical position is based on the original reference mapped position in the diploid genome sequences

calling of paralogous SNPs. The final step of remapping the oligonucleotide probe for each SNP assay back to the reference genomes and selecting those with the lowest copy number further facilitated the design of the array, since $81 \%$ of the SNPs that matched only once in the B. napus genome produced genome-specific three cluster patterns. Approximately $58 \%$ of all assays produced clear genomespecific genotype calls for a wide range of $B$. napus genotypes, including $F_{1}$ individuals. This is a marked improvement over similar arrays designed for hexaploid and tetraploid wheat where not more than $25.5 \%$ of the SNP assays produced clear genome-specific cluster patterns (Wang et al. 2014). However, the stringency of the design pipeline limited the variation available to be employed for the array design, effectively reducing the number of initial SNPs by $99 \%$. This high level of attrition could cause some marker selection bias. Thus, to achieve an optimal density of markers across the genome, pre-validated SNP assays, which matched two or more regions of the reference genome, were also included.

Upon testing $B$. napus cultivars originating from multiple continents and covering the range of annual and biennial types, the SNP assays proved to be highly polymorphic, with only $3.5 \%$ monomorphic loci identified indicating the value of the selected SNPs. This was also confirmed independently in a recently published work that used the array to assess diversity within a collection of predominantly Asian B. napus lines (Qian et al. 2014). The array was tested through the generation of a dense SNP map for B. napus with 21,766 (46\%) of the loci mapped in one DH population. Due to the design process, each of the SNP loci was physically anchored to a specific base-pair position in one or other of the diploid progenitor genomes (Supplementary Table 3). On extending this analyses to $B$. napus, however, the alignment of only the isolated short SNP regions to the genome sequence introduced a level of ambiguity, with some SNPs being equally likely to align, or in the case of the assays, hybridise to the $\mathrm{A}$ or $\mathrm{C}$ genome of $B$. napus. In addition, due to the prevalent homoeologous recombination events which have occurred during the evolution of B. napus (Chalhoub et al. 2014), it was expected that some SNPs may map to alternate orthologous positions in different $B$. napus genotypes. Comparing the genetic and physical position for each of the SNP loci, it was found that $3 \%$ of the loci were genetically mapped to a homoeologous region relative to their physically defined coordinates 


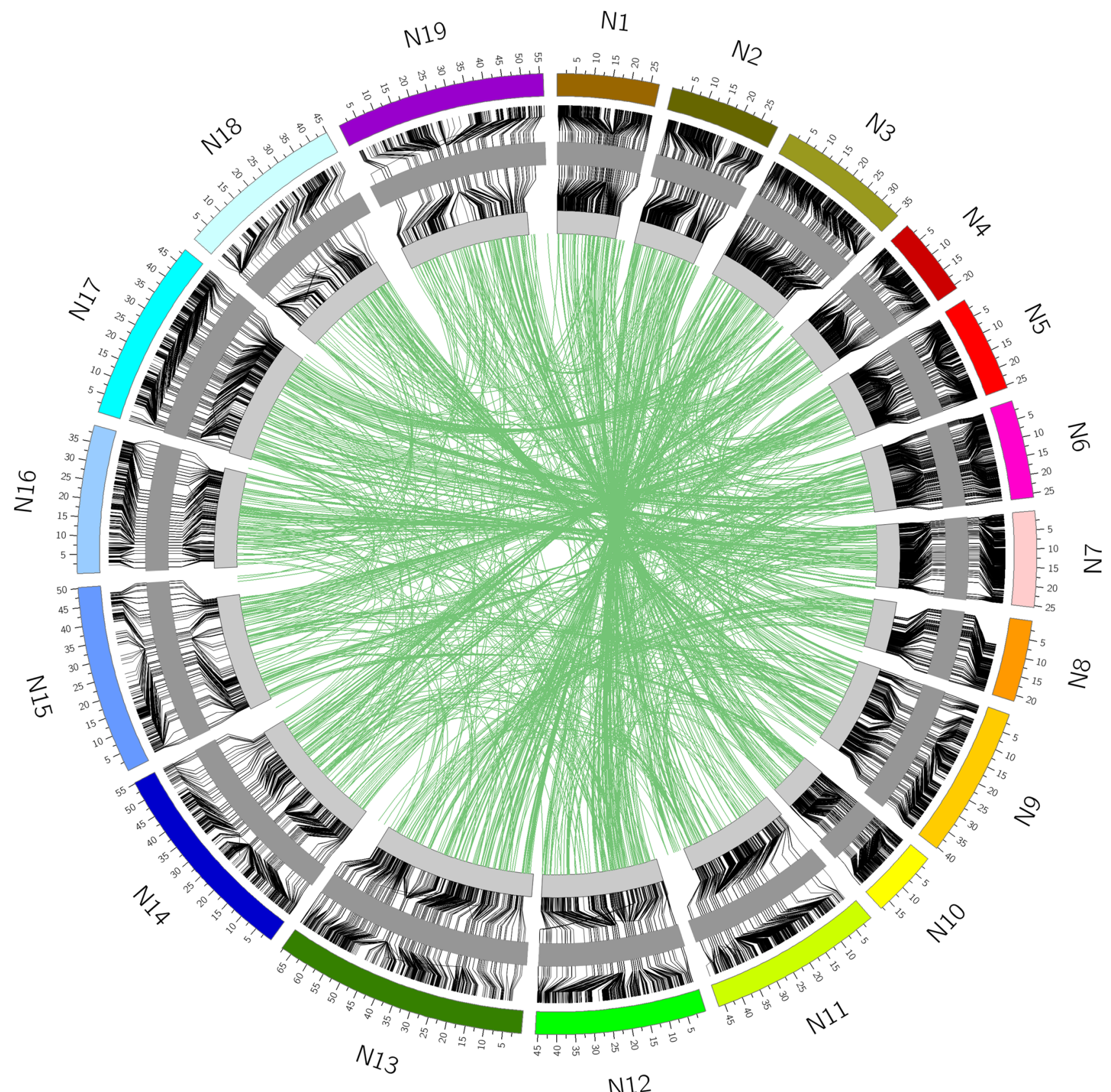

Fig. 4 Relationship between the physical and genetic positions of the SNP loci in B. napus. The inner circle represents the genetic map which is flanked to the outside by the physical position in the spring-type DH12075 and to the inside by the physical position in the

(Fig. 4). Further studies with the array are likely to uncover additional ambiguities that should be considered when utilising the array for analyses, especially when the loci cannot be genetically anchored in the population or specific genotypes being queried.

Although not specifically designed for this purpose, assessment of the array using DNA from the diploid winter-type Darmor bzh. The green lines connecting across the centre of the circle represent those loci that are genetically positioned to an alternate (mostly homoeologous) position compared to their physical coordinates in the genome sequence

genomes of B. oleracea and B. rapa demonstrated its value for genetic analysis of these two important vegetable crop species. Although, the genome specificity of many of the markers could be seen as a disadvantage, since those specific for the other genome result in failed assays, there are still 26,504 and 29,720 clearly scorable markers for $B$. oleracea and $B$. rapa, respectively. In addition, despite the 


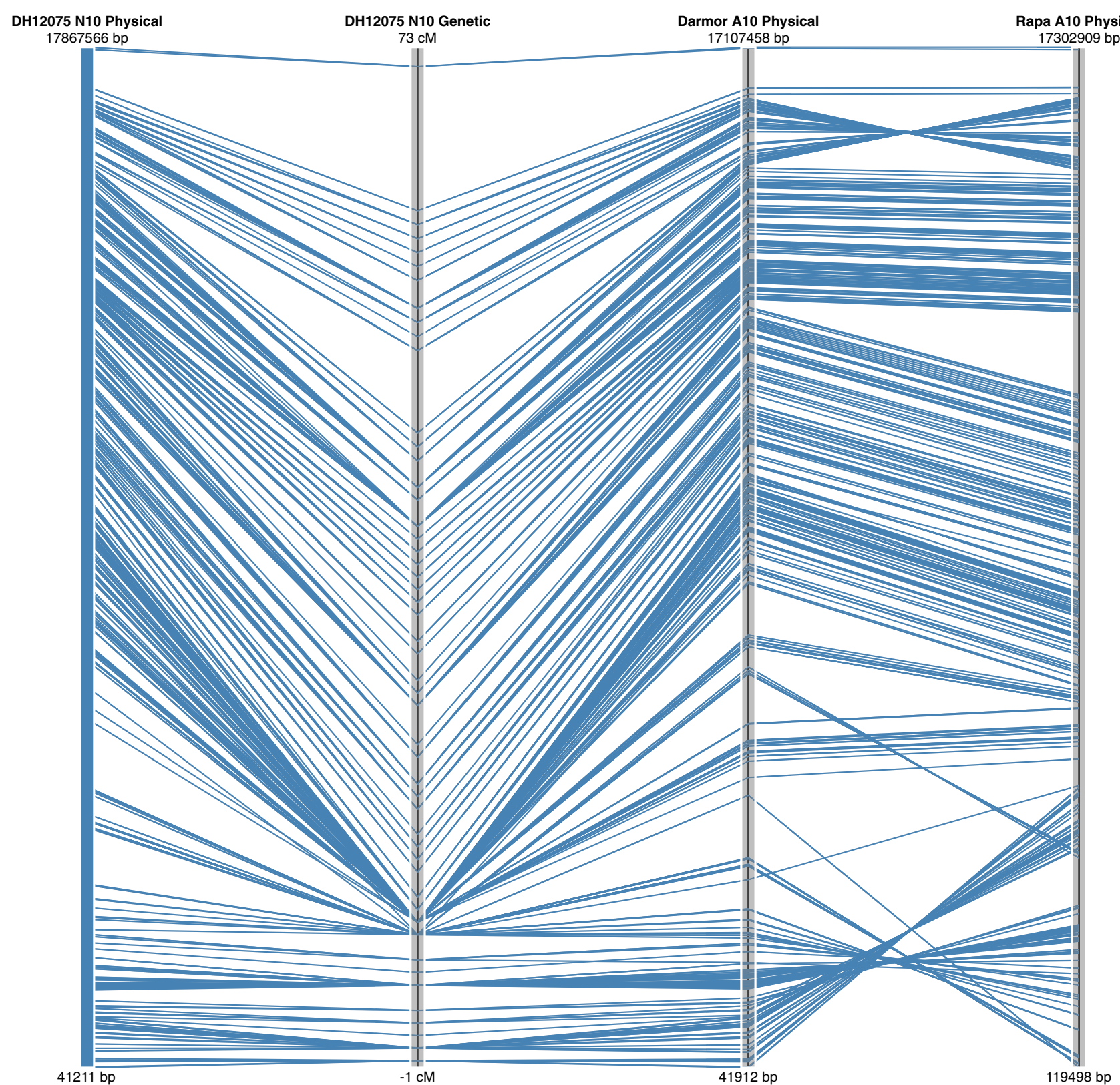

Fig. 5 Alignment of the genetic map for linkage group N10 of B. napus with the genome sequence of the equivalent chromosome in the two independent $B$. napus assemblies and in B. rapa

analysed sample number being lower, 21,113 and 22,695 markers were shown to be polymorphic in $B$. oleracea and B. rapa, respectively, demonstrating the utility of the array for these two diploid species. Indeed, this was confirmed through the recent generation of a genetic map for B. oleracea using the array (Brown et al. 2014).

Although the array offers relatively good coverage of the B. napus genome with the SNP loci physically distributed across each of the chromosomes at an approximate density of 1 marker every $15 \mathrm{~Kb}$ based on the diploid genome length, there was a significant sub-genome bias observed with a higher density in the A compared to the $\mathrm{C}$ genome (one marker every $11 \mathrm{~Kb}$ cf. $19 \mathrm{~Kb}$ ) (Table 2). The genetic map was based on a highly polymorphic cross allowing almost $50 \%$ of the SNP loci to be positioned on the $B$. napus genome. The genetic map showed only small gaps with five $\geq 9 \mathrm{cM}$. However, when considering the physical distribution of the mapped loci, each chromosome apart from N3 and N5 had at least one interval greater than $500 \mathrm{~Kb}$, these larger intervals were also biased to the $\mathrm{C}$ genome with $89 \%$ (140/158) of such intervals being localized to the $\mathrm{C}$ genome. This could reflect differing levels 
of genetic variation between the sub-genomes of B. napus, as observed by others (Delourme et al. 2013; Qian et al. 2014) or may suggest further optimisation of the array should focus on selection of $\mathrm{C}$ genome loci. Nevertheless, any bias in distribution of loci should be considered in downstream applications using the array. Once aligned to $B$. napus, this distribution did not change markedly and no large physical gaps were observed based on the overall marker selection (Fig. 3). The saturated coverage is partly a reflection of the genome organisation, with extensive blocks of repetitive elements largely limited to the pericentromeric regions. In addition, although $34.4 \%$ $(17,955)$ of the SNP loci fall within annotated genes, the array design did not focus on functional SNPs, which can bias the marker distribution. In maize, a similar high-density array was developed that targeted genic regions and even bearing in mind the greater genome size, there were significant gaps in the physical SNP coverage on many chromosomes of at least $1 \mathrm{Mb}$ per chromosome (Ganal et al. 2011). Alignment of the physical and genetic maps for B. napus showed good collinearity; however, a number of rearrangements were noted on comparison with the diploid genomes. Although, some of these can be attributed to artifacts of the genome assembly process in each species, the larger rearrangements that are common to the two B. napus genomes may indicate chromosomal changes that have occurred since the fusion of the two progenitor genomes in the B. napus nucleus (Fig. 5).

The Brassica $60 \mathrm{~K}$ Infinium array provides a robust and efficient tool for genetic studies in B. napus. Due to the natural and breeding bottlenecks created in modern Brassica germplasm much emphasis is now placed on capturing the wider allelic diversity within the species gene pool (Bus et al. 2011). Genome-wide association studies (GWAS) in a number of other crop species have suggested the value of such analyses for exploiting untapped variation to identify causative loci for key economic traits (Cook et al. 2012; Zhao et al. 2011). An essential prerequisite for GWAS is the ability to query genome-wide polymorphisms that are spaced, such that the analyses are not limited by the observable linkage disequilibrium (LD) in the species of interest. More recent estimates for B. napus suggest LD breakdown across the genome, ranging from 0.3 to $1.7 \mathrm{cM}$ (Delourme et al. 2013) and 0.25-2.5 Mb (Qian et al. 2014) with LD decaying more rapidly in the A genome. The distribution of SNP loci across the genome, which lie well within the current LD estimates, should facilitate the use of the array for GWAS or QTL mapping to identify genes underlying traits of interest. The demonstration of the utility of the array within $B$. napus as well as its diploid ancestors $B$. oleracea and $B$. rapa indicates that the developed array can be used in the entire crossing range of these three species, providing a valuable tool for Brassica breeding applications.
Author contribution statement WEC completed bioinformatics analyses to generate the array design. EEH, JP, and RW carried out experimental work to complete the cluster files. EEH created the genetic map. YK assisted with SNP data analyses. CS, RL, and FI-L generated data that were used in the SNP design. JB, DE, JM, JP, BL, WC, $\mathrm{ED}, \mathrm{SR}$, RJS and BS contributed SNP assays to the array design and were members of the consortium that agreed the array design. JB and JM also provided material for cluster file optimisation. CTL, AS, and MWG participated in study design, and helped edit the manuscript. All authors read and approved the manuscript. IAPP coordinated the design process, analysed data, and wrote the manuscript.

Acknowledgments The authors would like to acknowledge funding support from the Brassica SNP Consortium (Agriculture and AgriFood Matching Initiative Investment and industry partners). Research at TraitGenetics was supported in part by the Grant 0315639A from the Ministry of Education and Research (BMBF) and research in China was funded in part by National Basic Research and Development Program of China (2006CB101600).

\section{Compliance with ethical standards}

Conflict of interest The authors MWG, RW, and JP have competing commercial interests as members of TraitGenetics $\mathrm{GmbH}$ which is a company that offers marker development and analysis (including this array) for commercial purposes. This does not alter the authors' adherence to sharing data.

Open Access This article is distributed under the terms of the Creative Commons Attribution 4.0 International License (http://creativecommons.org/licenses/by/4.0/), which permits unrestricted use, distribution, and reproduction in any medium, provided you give appropriate credit to the original author(s) and the source, provide a link to the Creative Commons license, and indicate if changes were made.

\section{References}

Brown AF, Yousef GG, Chebrolu KK, Byrd RW, Everhart KW, Thomas A, Reid RW, Parkin IA, Sharpe AG, Oliver R, Guzman I, Jackson EW (2014) High-density single nucleotide polymorphism (SNP) array mapping in Brassica oleracea: identification of QTL associated with carotenoid variation in broccoli florets. TAG Theor Appl Genet Theoretische und angewandte Genetik 127:2051-2064

Bus A, Korber N, Snowdon RJ, Stich B (2011) Patterns of molecular variation in a species-wide germplasm set of Brassica napus. TAG Theor Appl Genet Theoretische und angewandte Genetik 123:1413-1423

Bus A, Hecht J, Huettel B, Reinhardt R, Stich B (2012) High-throughput polymorphism detection and genotyping in Brassica napus using next-generation RAD sequencing. BMC Genom 13:281

Chalhoub B, Denoeud F, Liu S, Parkin IAP, Tang H, Wang X, Chiquet J, Belcram H, Tong C, Samans B, Correa M, Da Silva C, Just J, Falentin C, Koh CS, Le Clainche I, Bernard M, Bento P, Noel B, Labadie K, Alberti A, Charles M, Arnaud D, Guo H, Daviaud C, Alamery S, Jabbari K, Zhao M, Edger PP, Chelaifa H, Tack D, 
Lassalle G, Mestiri I, Schnel N, Le Paslier MC, Fan G, Renault V, Bayer PE, Golicz AA, Manoli S, Lee TH, Thi VH, Chalabi S, Hu Q, Fan C, Tollenaere R, Lu Y, Battail C, Shen J, Sidebottom $\mathrm{CH}$, Wang $\mathrm{X}$, Canaguier A, Chauveau A, Berard A, Deniot G, Guan M, Liu Z, Sun F, Lim YP, Lyons E, Town CD, Bancroft I, Wang X, Meng J, Ma J, Pires JC, King GJ, Brunel D, Delourme R, Renard M, Aury JM, Adams KL, Batley J, Snowdon RJ, Tost J, Edwards D, Zhou Y, Hua W, Sharpe AG, Paterson AH, Guan C, Wincker P (2014) Plant genetics. Early allopolyploid evolution in the post-Neolithic Brassica napus oilseed genome. Science 345:950-953

Clarke WE, Parkin IAP, Gajardo HA, Gerhardt DJ, Higgins E, Sidebottom C, Sharpe AG, Snowdon RJ, Federico ML, Iniguez-Luy FL (2013) Genomic DNA enrichment using sequence capture microarrays: a novel approach to discover sequence nucleotide polymorphisms (SNP) in Brassica napus L. PLoS One 8:e81992

Cook JP, McMullen MD, Holland JB, Tian F, Bradbury P, Ross-Ibarra J, Buckler ES, Flint-Garcia SA (2012) Genetic architecture of maize kernel composition in the nested association mapping and inbred association panels. Plant Physiol 158:824-834

Dalton-Morgan J, Hayward A, Alamery S, Tollenaere R, Mason AS, Campbell E, Patel D, Lorenc MT, Yi B, Long Y, Meng J, Raman R, Raman H, Lawley C, Edwards D, Batley J (2014) A high-throughput SNP array in the amphidiploid species Brassica napus shows diversity in resistance genes. Funct Integr Genomics 14:643-655

Delourme R, Falentin C, Fomeju BF, Boillot M, Lassalle G, Andre I, Duarte J, Gauthier V, Lucante N, Marty A, Pauchon M, Pichon JP, Ribiere N, Trotoux G, Blanchard P, Riviere N, Martinant JP, Pauquet J (2013) High-density SNP-based genetic map development and linkage disequilibrium assessment in Brassica napus L. BMC Genom 14:120

Deschamps S, Llaca V, May GD (2012) Genotyping-by-sequencing in plants. Biology 1:460-483

Dixon GR (2006) Origins and diversity of Brassica and its relatives. Crop production science in horticulture 14. CABI, Wallingford, pp 1-33

Ganal MW, Altmann T, Roder MS (2009) SNP identification in crop plants. Curr Opin Plant Biol 12:211-217

Ganal MW, Durstewitz G, Polley A, Berard A, Buckler ES, Charcosset A, Clarke JD, Graner EM, Hansen M, Joets J, Le Paslier MC, McMullen MD, Montalent P, Rose M, Schon CC, Sun Q, Walter H, Martin OC, Falque M (2011) A large maize (Zea mays L.) SNP genotyping array: development and germplasm genotyping, and genetic mapping to compare with the B73 reference genome. PLoS One 6:e28334

Ganal MW, Polley A, Graner EM, Plieske J, Wieseke R, Luerssen H, Durstewitz G (2012) Large SNP arrays for genotyping in crop plants. J Biosci 37:821-828

Harper AL, Trick M, Higgins J, Fraser F, Clissold L, Wells R, Hattori C, Werner P, Bancroft I (2012) Associative transcriptomics of traits in the polyploid crop species Brassica napus. Nat Biotechnol 30:798-802

Iniguez-Luy F, Federico M (2011) The Genetics of Brassica napus. In: Schmidt R, Bancroft I (eds) Genetics and genomics of the brassicaceae. Springer, New York, pp 291-322

Jannink J-L, Lorenz AJ, Iwata H (2010) Genomic selection in plant breeding: from theory to practice. Brief Funct Genom 9:166-177

Kent WJ (2002) BLAT-the BLAST-like alignment tool. Genome Res 12:656-664

Lander ES, Green P, Abrahamson J, Barlow A, Daly MJ, Lincoln SE, Newberg LA (1987) MAPMAKER: an interactive computer package for constructing primary genetic linkage maps of experimental and natural populations. Genomics 1:174-181

Murray MG, Thompson WF (1980) Rapid isolation of high molecular weight plant DNA. Nucl Acids Res 8:4321-4325

Parkin IA, Clarke WE, Sidebottom C, Zhang W, Robinson SJ, Links MG, Karcz S, Higgins EE, Fobert P, Sharpe AG (2010) Towards unambiguous transcript mapping in the allotetraploid Brassica napus. Genome 53:929-938

Parkin IAP, Koh C, Tang H, Robinson SJ, Kagale S, Clarke WE, Town CD, Nixon J, Krishnakumar V, Bidwell SL, Denoeud F, Belcram H, Links MG, Just J, Clarke C, Bender T, Huebert T, Mason AS, Pires JC, Barker G, Moore J, Walley PG, Manoli S, Batley J, Edwards D, Nelson MN, Wang X, Paterson AH, King G, Bancroft I, Chalhoub B, Sharpe AG (2014) Transcriptome and methylome profiling reveals relics of genome dominance in the mesopolyploid Brassica oleracea. Genome Biol 15:R77

Qian L, Qian W, Snowdon RJ (2014) Sub-genomic selection patterns as a signature of breeding in the allopolyploid Brassica napus genome. BMC Genom 15:1170

Snowdon RJ, Iniguez Luy FL (2012) Potential to improve oilseed rape and canola breeding in the genomics era. Plant Breeding $131: 351-360$

UN (1935) Genome analysis in Brassica with special reference to the experimental formation of B. napus and peculiar mode of fertilization. Japan J Bot 7:389-452

Wang X, Wang H, Wang J, Sun R, Wu J, Liu S, Bai Y, Mun JH, Bancroft I, Cheng F, Huang S, Li X, Hua W, Wang J, Wang X, Freeling M, Pires JC, Paterson AH, Chalhoub B, Wang B, Hayward A, Sharpe AG, Park BS, Weisshaar B, Liu B, Li B, Liu B, Tong C, Song C, Duran C, Peng C, Geng C, Koh C, Lin C, Edwards D, Mu D, Shen D, Soumpourou E, Li F, Fraser F, Conant G, Lassalle $\mathrm{G}$, King GJ, Bonnema G, Tang $\mathrm{H}$, Wang $\mathrm{H}$, Belcram $\mathrm{H}$, Zhou H, Hirakawa H, Abe H, Guo H, Wang H, Jin H, Parkin IA, Batley J, Kim JS, Just J, Li J, Xu J, Deng J, Kim JA, Li J, Yu J, Meng J, Wang J, Min J, Poulain J, Wang J, Hatakeyama K, Wu K, Wang L, Fang L, Trick M, Links MG, Zhao M, Jin M, Ramchiary N, Drou N, Berkman PJ, Cai Q, Huang Q, Li R, Tabata S, Cheng S, Zhang S, Zhang S, Huang S, Sato S, Sun S, Kwon SJ, Choi SR, Lee TH, Fan W, Zhao X, Tan X, Xu X, Wang Y, Qiu Y, Yin Y, Li Y, Du Y, Liao Y, Lim Y, Narusaka Y, Wang Y, Wang Z, Li Z, Wang Z, Xiong Z, Zhang Z, Brassica rapa Genome Sequencing Project Consortium (2011) The genome of the mesopolyploid crop species Brassica rapa. Nat Genet 43:1035-1039

Wang S, Wong D, Forrest K, Allen A, Chao S, Huang BE, Maccaferri M, Salvi S, Milner SG, Cattivelli L, Mastrangelo AM, Whan A, Stephen S, Barker G, Wieseke R, Plieske J, International Wheat Genome Sequencing C, Lillemo M, Mather D, Appels R, Dolferus R, Brown-Guedira G, Korol A, Akhunova AR, Feuillet C, Salse J, Morgante M, Pozniak C, Luo M-C, Dvorak J, Morell M, Dubcovsky J, Ganal M, Tuberosa R, Lawley C, Mikoulitch I, Cavanagh C, Edwards KJ, Hayden M, Akhunov E (2014) Characterization of polyploid wheat genomic diversity using a high-density 90000 single nucleotide polymorphism array. Plant Biotechnol J 12:787-796

Wu Y, Bhat PR, Close TJ, Lonardi S (2008) Efficient and accurate construction of genetic linkage maps from the minimum spanning tree of a graph. PLoS Genet 4:e1000212

Zhao K, Tung CW, Eizenga GC, Wright MH, Ali ML, Price AH, Norton GJ, Islam MR, Reynolds A, Mezey J, McClung AM, Bustamante CD, McCouch SR (2011) Genome-wide association mapping reveals a rich genetic architecture of complex traits in Oryza sativa. Nat Commun 2:467 